\title{
Ponencia: Estudiantes
}

\section{Pedagogía universitaria y desenvolvimiento del estudiante: Un paradigma evidenciado en la capacidad de manejo del poder}

\author{
Luis Fernando Gutiérerrez Sáenz
}

\begin{abstract}
Resumen: La declaración de principios, propósitos y funciones de la Universidad de Costa Rica, estipulada en el Estatuto Orgánico, resalta el papel formativo de institución autónoma superior, con una concepción de estudiante, como proyecto humano integral de naturaleza biopsicosocial que crece, se desarrolla, actúa y transforma.

Como institución pública, la Universidad de Costa Rica tiene una misión social que cumplir. Consiste en formar profesionales con un sentido crítico, es decir, comprometidos con la vigencia de los derechos humanos en el ejercicio del poder. Este ejercicio ya mismo en su cotidianidad de aula y de vida universitaria, contribuye a la socialización de las emociones y reconstrucción de los modelos sociales que al practicarlos, también se modifican.

De acuerdo con los aspectos anteriormente citados y en el marco del Simposio Internacional de Pedagogía Universitaria, su filosofia y objetivos, se considera fundamental redimensionar en estas reflexiones la importancia de una participación real de los estudiantes en la definición, construcción y manejo del poder, como parte de su formación académico-profesional.
\end{abstract}

\section{Presentación}

La Educación, en un Estado de Bienestar y Desarrollo Sostenible, desempeña una función política consistente en orientar permanentemente la conservación, desarrollo y proyección de los grupos humanos, para el crecimiento y creación de la cultura nacional.

La Educación como proceso es formación integral y mediación, no enseñanza, destacaba Mejía (1978, p. 5). En correspondencia, la Pedagogía como epistemología ha de promover el desarrollo de la condición humana en sus creaciones culturales a partir de la emancipación y unicidad de los significados individuales, dinamizados por la interpersonalidad en sus relaciones.

Las universidades, en calidad de centros de educación superior, tienen la función principal de formar los investigadores que ansíen el cambio social (Villarroel, 1995). A ellos y ellas como profesionales les corresponden las funciones de construir, expresar y administrar el objeto de conocimiento, desde los campos de actividad derivados de las carreras y especialidades de las que se gradúan.

Dado el carácter personológico, social, estratégico y vinculante de la universidad, 
a la Pedagogía Universitaria le corresponde sistematizar, como parte del proceso de aprendizaje, las instancias normativas e integrar los recursos y servicios que la institución crea y sostiene para favorecer el desarrollo integral de los estudiantes en su aspecto formativo.

De este modo, se estimula en el estudiantado el talento, creatividad, inventiva y actitud en la concepción y producción de las propuestas personales, propiciando la ambientación de los espacios en el aula, en las prácticas de comunidad y laborales, así como en la vida universitaria, de modo tal que se provoque el desenvolvimiento emancipatorio de los jóvenes ante los problemas y soluciones desde la cotidianidad de su aprendizaje. Esto potencializa el desarrollo y crecimiento personales para el manejo del poder.

En correspondencia con los objetivos del Simposio interesa dar a conocer de manera resumida los resultados de investigaciones cualitativas en el manejo de la normativa universitaria por parte de estudiantes, docentes y administrativos. Todo ello tal como aparece en el documento "Conozcamos los reglamentos estudiantiles" elaborado y presentado con ocasión del Foro Internacional "Nuestros Retos Globales", organizado por el Ministerio de Relaciones Exteriores y Culto, Universidad para la Paz y Fundación Milenio; en noviembre 2000.

Para este efecto, en las siguientes páginas, el tema se trabajará en el siguiente orden: El problema y su importancia; El modelo paradigmático o La Utopía; La metodología de trabajo; Resultados obtenidos y Alternativas de acción.

\section{El problema y su importancia}

Vivir, existir, expresarse, resolver, enfrentar situaciones, son acciones de logro que implican procesos, estilos y objetivos de actuación, los que varían según las diferencias y significados para cada sujeto. Estas conductas van conformando los valores culturales que luego se transfieren, forjando patrones de conducta y aprendizaje social.

Los centros educativos en Costa Rica, cuya competencia gira en la formación de los recursos humanos, entienden por evaluación de los aprendizajes la sola medición del eje de los contenidos (Sequeira, 1992). Esta condición pedagógica provoca una escisión en el sujeto del aprendizaje, ya que al centrarse este en el dominio del contenido, no se trabaja integralmente el componente personológico y, más lamentable aún, no se llega en forma directa ni congruente hasta el objetivo o misión de la educación, la cual es la mejora o cambio permanente en las condiciones de vida.

Esta realidad a lo largo de los años, ha contribuido a acentuar, en los integrantes de los procesos de aprendizaje, el desarrollo o reproducción de actitudes y valores que automatizan una reflexividad con respuesta simple, convencional, lógica, pasiva, pero también individualista, dependiente, autoritaria y abusiva; reforzada por situaciones humanas que se tienen que enfrentar cotidianamente y en las que se pretende llegar a soluciones constructivas, pero que no se logran.

A propósito de lo anterior, un estudio sobre estilos de aprendizaje en una muestra de estudiantes de tercer año de las carreras de la Universidad de Costa Rica (Gutiérrez, 2000) arrojó para todas las áreas académicas una tendencia de "muy alta" a "alta" en la composición del pensamiento "convergente" conformado, en términos de Alonso (1995), por los estilos teórico (exceptuando Ciencias Básicas) y pragmático (exceptuando Artes y Letras). Con respecto al pensamiento "divergente", la tendencia de los estilos activo y reflexivo fue moderada en ambos, con excepción de Letras e Ingeniería. 
Un estudio sobre el mismo tema dirigido por Flory Estela Bonilla en el año de 1988, ya había obtenido resultados similares, lo cual indica que en veinte años la Universidad no ha logrado un cambio cualitativo en la formación integral de los estudiantes.

Por el contrario, cuando se comprende que estos rasgos son condiciones en constante surgimiento como ingredientes de los procesos de vida en los mismos espacios de aprendizaje, las aulas se convierten en los laboratorios de investigación y práctica, para dar y manejar soluciones creativas, constructivas y de consenso, las cuales se propician cuando las relaciones entre los actores son "bilaterales".

En la Universidad de Costa Rica, aquellas prácticas tradicionales han provocado e intensificado una incapacidad en el manejo del poder por parte de los integrantes del proceso de aprendizaje, por ejemplo, a la hora de enfrentar la dificultad de los contenidos, así como de los componentes o factores influyentes, tanto de los cursos en general, como de aquellos denominados "cursos no ponderables", o bien en la participación de los estudiantes en el planteamiento y manejo de los procedimientos ante diversas instancias; o la evaluación de los aprendizajes, que se permite prescindir de todo lo anterior.

Esto repercute en el objeto del conocimiento, preparación y actuación, como parte de la formación académica profesional de los estudiantes. Como contraparte, también se fomentan la división y el individualismo en los educandos, así como la incomunicación en las relaciones entre autoridades, lo cual dificulta, cuando no aniquila, la construcción de los procedimientos-objetivo que son los que favorecen procesos más eficientes, ágiles y flexibles.
Hipótesis. La formación de futuros profesionales, en un mundo globalizado y en contextos de cambio, compromete la participación activa de los procesos de aprendizaje, de modo tal que, toda situación derivada de la construcción de los aprendizajes entre los actores, en vez de afectar las relaciones humanas con actitudes autoritarias de intolerancia o de condescendencia de las partes, se resocialicen como elementos de aprendizaje del poder. Esto facilita el ejercicio de la tolerancia, así como de la participación ciudadana, en la resolución creativa y oportuna de los problemas de los actores.

\section{El modelo paradigmático o la utopía}

La declaración de principios, propósitos y funciones de la Universidad de Costa Rica, estipulada en el Estatuto Orgánico, resalta el papel formativo de institución autónoma superior, con una concepción de estudiante, como proyecto humano integral de naturaleza biopsicosocial que crece, se desarrolla, actúa y transforma.

Como institución pública, la Universidad de Costa Rica tiene una misión social que cumplir. Consiste en formar profesionales con un sentido crítico, es decir, comprometidos con la vigencia de los derechos humanos en el ejercicio del poder. Este ejercicio ya mismo en su cotidianidad de aula y de vida universitaria, contribuye a la socialización de las emociones y reconstrucción de los modelos sociales que al practicarlos, también se modifican.

La universidad es entonces, un espacio de encuentro de culturas de "vida". Esto es, de vivencia humana permanente y cotidiana, donde los estudiantes interactúan en los espacios universitarios orientados por sus objetivos, estrategias de aprendizaje en 
el estudio, la investigación, la meditación, la experimentación, el debate o defensa de ideas en la participación y la puesta en práctica de las propuestas programáticas de cada curso, cuando son transformadas por los equipos de trabajo estudiantil.

En correspondencia, el estudiante, en tanto tiene la capacidad transformadora, garantiza en sus acciones el compromiso de la práctica creativa de sus derechos en coherencia con los valores de igualdad, justicia, cooperación, respeto y solidaridad. Tales derechos, al apropiarse primeramente en la relación del estudiante con el grupo de pertenencia, se extienden después con facilidad a los espacios de proyección social, cumpliendo así encuentros permanentes entre los intereses de la institución con los intereses del estudiantado y de los beneficiarios en la sociedad donde él o ella interactúan.

En un modelo educativo para la transformación, los procesos de aprendizaje constituyen una cultura de diálogo, comunicación, respeto, responsabilidad y ética. El reconocimiento de los actores como "consocios" (Gutiérrez, 2000) establece una serie de principios, valores, actitudes y experiencias, enmarcadas en sus proyectos de vida. Esto facilita construir el conocimiento para la acción.

Al consolidar esta relación, se requiere que cada actor tenga claridad y responsabilidad de sus funciones ante la construcción del proceso educativo, el cual tiene una implicación intelectual y emocional de estudio y análisis de los aprendizajes, que se expresa en el desenvolvimiento de los estudiantes en el dominio del conocimiento, así como en el manejo de su realidad en el ámbito educativo y en el ejercicio del poder de cada curso y programa; así como en los espacios de la administración curricular instancias y servicios.
De tal modo, se generan metodologías que propician una participación activa, crítica y democrática de los sectores involucrados y un compromiso activo en el propio desenvolvimiento y cambio. Esto significa reconocer que, cada actor del proceso formativo está sujeto a derechos y deberes, lo cual los lleva a reconocer también la propia identidad en el proceso.

La enseñanza y el aprendizaje constituyen entonces un solo proceso, cuyo fin es el desarrollo de habilidades y actitudes de los participantes desde sus intereses y valores, en convivencia con las metas institucionales, incluyendo la sociedad en su conjunto. Aquí, el docente tiene un papel fundamental como facilitador del proceso de aprendizaje de los estudiantes y la evaluación un carácter integrador.

La experiencia histórica de la diversidad de economías, así como los acontecimientos globales, vienen demostrando que la capacidad de convivencia humana demanda una educación que la haga posible.

\section{Metodología de trabajo}

En el contexto de la Universidad de Costa Rica, el quehacer de los diversos actores, así como el proceso de aprendizaje, están normados en una legislación que regula los derechos y los deberes, los recursos y los procedimientos.

En correspondencia con lo anterior y el propósito ${ }^{1}$ de la Oficina de Orientación de la Vicerrectoría de Vida Estudiantil, se han desarrollado distintas estrategias individuales y grupales dirigidas a estudiantes, así como a funcionarios docentes, administrativos y autoridades universitarias, tendientes a socializar información de conceptos, normas, y procedimientos para prevenir situaciones 
que podrían convertirse en factores de riesgo, para los objetivos de la formación universitaria.

\section{Resultados}

1. En la cotidianidad de la vida universitaria y del quehacer de los equipos interdisciplinarios que conforman la Oficina de Orientación de la Vicerrectoría de Vida Estudiantil, se han identificado situaciones que ponen en evidencia, a partir de los resultados obtenidos, un desconocimiento generalizado de la normativa universitaria.

2. En lo específico, el Reglamento de Régimen Académico Estudiantil dirige y norma el proceso de aprendizaje universitario. Sin embargo, en la práctica, se observa que hay desconocimiento del modelo educativo estatutario, al centrar el objeto de estudio y evaluación del aprendizaje en los contenidos temáticos sin contextualizar, integrar o apreciar el desenvolvimiento del estudiante desde sus conocimientos previos, sus estilos de aprendizaje, sus preferencias vocacionales y sus dificultades académicas como parte de su realidad cotidiana, integrados a la vez en los procesos de aprendizaje.

3. Por otro lado, la docencia, al hacer la presentación y análisis de sus propuestas programáticas en cada inicio de ciclo lectivo, es frecuente observar que la estructura de los programas de curso, no especifican las cualidades del perfil profesional que se pretende desarrollar en cada uno de éstos. En consecuencia, la evaluación no integra lo que los estudiantes aportan desde sus objetivos, intere- ses y recursos personológicos (Mitjans, 1995), para conformar el programa de curso. No obstante, los docentes sí formalizan en este momento las relaciones de poder a las que se induce al estudiante.

4. En contraste, también es evidente en algunos cursos la subjetividad, el irrespeto, o aún el mismo concepto de estudiante y de educación que se tiende a identificar, en el proceso educativo. Este responde a un modelo que es sinónimo de transmisión de información, lo que forma individuos pasivos, temerosos de la autoridad e incapaces de enfrentar la diferencia, la disensión y el conflicto.

5. En otro orden de cosas, se han encontrado situaciones como las siguientes: ser vocero de inconformidad desde lo que el estudiante considera respecto al sistema de evaluación de un curso, el irrespeto a la condición de estudiante-persona, el incumplimiento por parte del docente en algunas partes del proceso determinado por algún artículo de los que establece el reglamento antes mencionado.

En razón de lo anterior, al estudiante que evidencia alguna situación anómala, "curiosamente" se le señala como "estudiante problema", "estudiante vago". Además, tiene que encarar una serie de procedimientos administrativos burocráticos que le provocan frustración, resentimiento, agresión hasta claudicar del trámite que había iniciado para la resolución del conflicto, escuchándose frases como: "para qué apelar un examen si no me van a arreglar la nota", "no puedo denunciar el profesor que me hostiga porque me va a reprobar" "me van a señalar si hablo y esto me 
perjudicaría para graduarme”. En situaciones extremas también proliferan hasta los recursos de "Sala Cuarta".

El proceso de aprendizaje desde una perspectiva de contenidos, también genera evaluaciones sumativas que promueven la reproducción de conocimiento, lejos de prepararlos para enfrentar el medio social con acciones estratégicas o soluciones creativas y emprendedoras. En este sentido Yarzábal (1999), plantea que los centros de Educación Superior, tienen un efecto determinante en el contexto social que contribuye o no en una dirección eficiente y una orientación para el desarrollo.

De acuerdo con los aspectos anteriormente citados y en el marco del I Simposio Internacional de "Pedagogía Universitaria", su filosofía y objetivos, se considera fundamental redimensionar en estas reflexiones la importancia de una participación real de nuestra población estudiantil en formación académico-profesional desde su identidad, valores, historias de vida y su papel como agentes de cambio en la definición, construcción y manejo del poder como condición sine qua non de los procesos de aprendizaje.

\section{Alternativas de acción}

1. Los programas de curso son contratos de aprendizaje. Como tales, tienen una composición técnica, académica y legal que compromete el desarrollo del pensamiento de los participantes de acuerdo con los objetivos, contenidos, metodología y evaluación que se proponen. A la vez, constituyen el documento legal que acredita al educando para el reconocimiento de cursos en otra carrera o universidad. Finalmente, la suma de los programas de cursos componen el plan de estudios. Éstos, aprobados por los estudiantes, los acreditan para obtener el título correspondiente. Por lo tanto:

- Que los programas de curso contemplen en el apartado que consigna la evaluación de curso la siguiente leyenda "el proceso de aprendizaje en todos sus extremos se regula por lo que establece el Reglamento de Régimen Académico Estudiantil".

- Que los programas de curso contemplen en forma visible desde los objetivos y actividades que se proponen:

1. Las habilidades, destrezas, actitudes y valores básicos que se pretende o se espera que cada estudiante desarrolle o fortalezca.

2. Las técnicas y herramientas que se pondrán en práctica.

3. La evaluación incluya la modalidad formativa, evaluando el desenvolvimiento de los estudiantes en el manejo o dominio de las técnicas, las herramientas $\mathrm{y}$ otras estrategias novedosas, según el nivel de dificultad del curso en el programa de estudios.

4. Un resumen del curriculum básico del docente(s) que imparte el curso.

2. Que cada curso incluya una apreciación del curso al término de éste, hechas por los estudiantes y el docente y se le de seguimiento a los resultados.

3. Que en las dos primeras semanas de inicio del curso, de acuerdo con los artículos 13 y 15 del R.R.A.E. el grupo de estudiantes se aboque a un análisis del programa de curso adecuando una metodología que propicie la participación y aporte de los estudiantes en la elaboración de sus propuestas de trabajo. Todo lo cual permitirá al docente identificar las diferencias 
individuales que determinarán el estilo de desenvolvimiento de los estudiantes.

4. Que se destine a uno de los cursos introductorios del primer año de carrera, el espacio para el estudio y análisis formativo de la normativa universitaria estudiantil de modo tal que se promuevan alternativas de acción para el manejo y solución de situaciones. Todo esto de acuerdo con las características de los estudiantes, del sistema y particularidades de la carrera y unidad académica.

5. Que los cursos específicos del primer año de las carreras se asignen a docentes reconocidos por la unidad académica por su capacidad y estilos de interrelación social que favorecen el clima de aprendizaje. Preferiblemente con algunas de las siguientes características según $\mathrm{Gu}$ tiérrez (2000):

- Cualidades: tales como: apertura, afablilidad que facilita la comunicación con el estudiante, atento ante las diferencias individuales y las necesidades del grupo, paciente, líder con autoridad y que promueven el uso de metodologías participativas.

- Valores: disponiblidad, honestidad, calidad humana, responsabilidad, ética, sentido de superación.

- Formación profesional: actualizado con dominio de su área, estrategias didácticas, capacidad de investigación, gusto por su especialidad, estimula el conocimiento, objetividad en la evaluación.

6. Que especialmente en el primero y tercer año de carrera, los profesores consejeros se integren como agentes de apoyo en el desarrollo de los estudiantes aportando críticamente en el trámite y procedimientos administrativos, así como en el enriquecimiento de los aprendizajes ante los análisis y planteamientos de los estudiantes.

7. Que durante la carrera y de acuerdo con la respuesta de los estudiantes en sus procesos de aprendizaje se promuevan talleres orientados a fortalecer el autoconcepto y la autoestima de los estudiantes en el marco crítico de lo que proponen los principios del Estatuto Orgánico y la normativa universitaria estudiantil.

8. Que las conductas, actitudes, valores, habilidades que se derivan de la normativa universitaria estudiantil se integre en los componentes curriculares y se reflejen en cada curso y programa como ingrediente de la formación académico profesional del estudiantado.

9. Que el contenido de los reglamentos estudiantiles forme parte de la inducción a los docentes que ingresan al sistema.

\section{Nota}

1. "Promover un proceso de orientación integral para ayudar al estudiante en la construcción de respuestas a necesidades que surgen durante su admisión, permanencia y graduación”. Plan de Trabajo 2000. Oficina de Orientación. Vicerrectoría de Vida Estudiantil. Universidad de Costa Rica.

\section{Referencias bibliográficas}

Bonilla, Fl. "Estilos de aprendizaje de los estudiantes de la Universidad de Costa Rica". En: Revista Educación de la Universidad de Costa Rica. 12 (1), pp. 17-26. 1988.

Gutiérrez L. Estilos de aprendizaje en una muestra de estudiantes que estudian la carrera de su interés en 
Universidad de Costa Rica. Seminario de investigación del programa de maestría en Psicopedagogía. Universidad Estatal a Distancia. 2000.

Gutiérrez L. E. Iturrino M. Conozcamos los reglamentos estudiantiles: identidad, valor y acción para transformar. Oficina de Orientación. Vicerrectoría de Vida Estudiantil. Universidad de Costa Rica. 2000.

Mejía, H. "El Ser de la Educación”. En: Revista Educación de la Universidad de Costa Rica. II (2), pp. 1-9. 1978.
Mitjáns, A. Creatividad, Personalidad y Educación. Madrid. Editorial Pueblo y educación. 1995.

Sequeira, A. "Perspectivas curriculares para el siglo XXI”. En: Gurdián, A. (1994) Proyecto de Universidad para el Siglo XXI: Cátedra Emma Gamboa. Universidad de Costa Rica. Oficina de Publicaciones. San José. 1992.

Yarzábal, L. Consenso para el Cambio. Instituto Internacional para la Educación Superior en América Latina y el Caribe. IESALC/UNESCO. Caracas. 1999. 